Research Article

\title{
On Progressive Type-II Censored Samples from Alpha Power Exponential Distribution
}

\author{
Mukhtar M. Salah \\ Department of Mathematics, College of Science, Majmaah University, Al Majmaah 11952, Saudi Arabia \\ Correspondence should be addressed to Mukhtar M. Salah; m.salah@mu.edu.sa
}

Received 23 August 2020; Revised 13 September 2020; Accepted 15 October 2020; Published 16 November 2020

Academic Editor: Hijaz Ahmad

Copyright (C) 2020 Mukhtar M. Salah. This is an open access article distributed under the Creative Commons Attribution License, which permits unrestricted use, distribution, and reproduction in any medium, provided the original work is properly cited.

In this paper the two-parameter $\alpha$-power exponential distribution is studied. We study the two-parameter $\alpha$-power exponential $(\mu, \lambda)$ distribution with the location parameter $\mu>0$ and scale parameter $\lambda>0$ under progressive Type-II censored data with fixed shape parameter $\alpha$. We estimate the maximum likelihood estimators of these unknown parameters numerically since it cannot be solved analytically. We use the approximate best linear unbiased estimators $\mu^{*}$ and $\lambda^{*}$, as an initial guesses to obtain the MLEs $\widehat{\mu}$ and $\hat{\lambda}$. We estimate the interval estimation of these unknowns' parameters. Monte Carlo simulations are performed and data examples have been provided for illustration and comparison.

\section{Introduction}

The censoring sample takes place in the life testing experiment, if we cannot notice the failure time of all items placed on this experiment. To see this, assume a life-testing experiment consisting from $s$ units was kept under observation, and these units may be in industry manufactory, individuals in clinical queue, computers, and any system in a reliability study experiments so that if we preplanned to remove some units from this experiment in order to provide saving cost and time then after removing such units from this experiment, the resulting data are named censored data. There are three types of censoring schemes: Type-I, Type-II, and progressive censoring schemes. Before defining these types, assume $n$ items are kept on a testing-life experiment; then,

(1) Assume we decided to stop the experiment at prefixed time $T$, to get only the failure time of the items which fails prior to the time recorded, and the resulting sample from this experiment is defined as Type-I censoring sample.

(2) Assume that an experiment is stopped at $R$ th failure then the resulting data is defined as Type-II censored sample; note here $R$ th failure is considered to be fixed, while $X_{R: n}$ denotes a random experiment duration. For more details about Type-I and Type-II censored samples, one can refer to Salah [1], Balakrishnan and Aggarwala [2], Pradhan and Kundu [3], and Lin et al. [4].

(3) Progressive censoring sample: assume $s$ identical items are placed on a life-testing experiment, and it is decided to observe only $k$ failures, and censor the remaining $s-k$ items progressively as follows. At the time of the first failure, $F_{1}$ of the $(s-1)$ surviving components are removed randomly from the experiment; at the time of the next failure, $F_{2}$ of the remaining $\left(s-2-F_{1}\right)$ surviving components are removed randomly from the experiment; repeat this censoring up to the final stage, the time of the $k$ th failure, and we have all remaining $F_{k}=s-k-\left(F_{1}-F_{2}-\cdots-F_{k-1}\right)$. The surviving components are randomly removed from the experiment and then the resulting censoring is called progressive censoring. More precisely, it is named as progressive Type-II censoring sample.

Let the sample $Z_{1: k: s}, Z_{2: k: s}, \ldots, Z_{k: k: s}$ be a progressively Type-II censored sample and $\left(F_{1}, F_{2}, \ldots, F_{k}\right)$ be the progressive censoring scheme. Suppose that $k$, the number of 
observed failures, and $\left(F_{1}, F_{2}, \ldots, F_{k}\right)$ are all prefixed. Then, we have the following special cases:

(1) If $F_{1}=F_{2}=\cdots=F_{k-1}=0$ and $F_{k}=s-k$, then this scheme is reduced to the Type-II censoring scheme.

(2) If $F_{1}=F_{2}=\cdots=F_{k}=0$ and $k=s$, then no censoring happens (the complete data case). For progressive censoring and its inferences, one can refer to Balakrishnan [5], Balakrishnan et al. [6], Salah [7], Salah [8], Salah [9] and Khan et al. [10].

Mahdavi and Kundu [11] present a new method to add a new parameter to a family of distribution. They named it as $\alpha$ - power transformation (APT) method. They applied the APT method to a specific class of distribution such as the exponential distribution, and they called this new distribution as the two-parameter $\alpha$-power exponential (APE) distribution. In this paper, we study the statistical inferences of the $\operatorname{APE}(\mu, \lambda)$ distribution under progressive Type-II censoring schemes. Here, we define the APE distribution as follows.

Definition 1. Let $X$ be a random variable, then $X$ follows a two-parameter APE distribution $\operatorname{APE}(\mu, \lambda)$, with location parameter $(\mu>0)$ and scale parameter $(\lambda>0)$ if the probability density function (pdf) and the cumulative distribution function (cdf) of $X$ for any $x>0$, respectively, are

$$
\begin{aligned}
& f(x ; \mu, \lambda)= \begin{cases}\frac{\log \alpha}{\alpha-1} \lambda e^{-\lambda(x-\mu)} \alpha^{1-e^{-\lambda(x-\mu)},} & \text { if } \alpha \neq 1, \\
\lambda e^{-\lambda(x-\mu)}, & \text { if } \alpha=1,\end{cases} \\
& F(x ; \mu, \lambda)= \begin{cases}\frac{\alpha^{1-e^{-\lambda(x-\mu)}-1}}{\alpha-1}, & \text { if } \alpha \neq 1, \\
1-e^{-\lambda(x-\mu)}, & \text { if } \alpha=1 .\end{cases}
\end{aligned}
$$

The rest of this paper is organized as follows. In Sections 2 and 3 , we present the maximum likelihood estimators MLEs $(\widehat{\mu}, \widehat{\lambda})$ and Fisher information matrix to estimate the interval estimation for $\mu$ and $\lambda$. In Section 4 , we derive the approximate best linear unbiased estimators ABLUEs $\left(\mu^{*}, \lambda^{*}\right)$ for $\mu$ and $\lambda$. In Section 5, numerical calculation and simulation are preformed. Finally, conclusion is given in Section 6.

\section{Maximum Likelihood Estimator}

This section presents the MLE $(\widehat{\mu})$ of $\mu$ and the MLE $(\hat{\lambda})$ of $\lambda$ from the $\operatorname{APE}(\mu, \lambda)$ distribution when the samples are progressively Type-II censored. To this end, assume $\underset{\sim}{X}=$ $\left(X_{1: m: n}, X_{2: m: n}, \ldots, X_{m: m: n}\right)$ is a progressive Type-II censoring sample from APE distribution and $\left(R_{1}, R_{2}, \ldots, R_{m}\right)$ is the progressively censoring scheme. Then, we have likelihood function $L(\underset{\sim}{X}, \mu, \lambda)$ regarded to progressive Type-II censored sample as

$$
L(\underset{\sim}{X}, \mu, \lambda)=c \prod_{i=1}^{m} f\left(x_{i: m: n}, \mu, \lambda\right)\left[1-F\left(x_{i: m: n}, \mu, \lambda\right)\right]^{R_{i}},
$$

where

$$
\begin{aligned}
c= & n\left(n-1-R_{1}\right)\left(n-2-R_{1}-R_{2}\right), \ldots, \\
& \cdot\left(n-m+1-R_{1}-R_{2}-\cdots-R_{m-1}\right) .
\end{aligned}
$$

For simplicity, we use $L$ instead of $L(\underset{\sim}{X}, \mu, \lambda)$ and $x_{i}$ for $x_{i: m: n}$. Now, by substituting equations (1) and (2) into equation (3) with some simplifications, we obtain

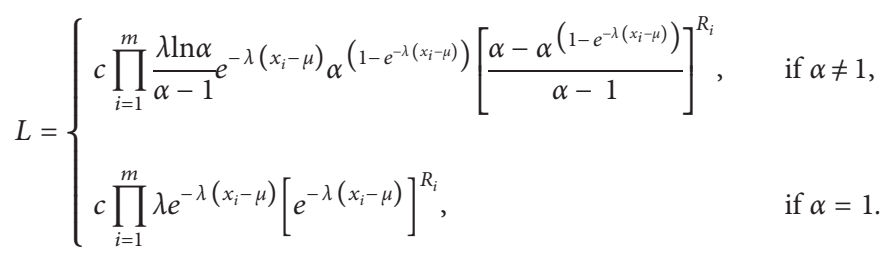

Since when $\alpha=1$ in equation (5), then we have the loglikelihood for the exponential distribution which is a special case from APE distribution and is studied before, one can refer to Balakrishnan et al. [6]. Now, from equation (5), the log-likelihood function is given by

$$
\ln L=\ln c+m \ln \frac{\lambda \ln \alpha}{\alpha-1}-\sum_{i=1}^{m} \lambda\left(x_{i}-\mu\right)+m \ln \alpha-\ln \alpha \sum_{i=1}^{m} e^{-\lambda\left(x_{i}-\mu\right)}+\sum_{i=1}^{m} R_{i}\left[\ln \left(\alpha-\alpha^{\left(1-e^{-\lambda\left(x_{i}-\mu\right)}\right)}\right)-\ln (\alpha-1)\right]
$$


Differentiating equation (6) with respect to $\mu$ and $\lambda$, respectively, we obtain

$$
\frac{\partial \ln L}{\partial \mu}=m \lambda-\lambda \ln \alpha \sum_{i=1}^{m} e^{-\lambda\left(x_{i}-\mu\right)}+\lambda \ln \alpha \sum_{i=1}^{m} \frac{R_{i} e^{-\lambda\left(x_{i}-\mu\right)}}{\alpha^{e^{-\lambda\left(x_{i}-\mu\right)}}-1},
$$

$$
\begin{aligned}
\frac{\partial \ln L}{\partial \lambda}= & \frac{m}{\lambda}+\sum_{i=1}^{m}\left(x_{i}-\mu\right)\left(\ln \alpha e^{-\lambda\left(x_{i}-\mu\right)}-1\right) \\
& -\ln \alpha \sum_{i=1}^{m} \frac{R_{i}\left(x_{i}-\mu\right) e^{-\lambda\left(x_{i}-\mu\right)}}{\alpha^{e^{-\lambda\left(x_{i}-\mu\right)}}-1} .
\end{aligned}
$$

The MLEs $\widehat{\mu}$ and $\hat{\lambda}$ can be estimated by solving system of equations: $(\partial \ln L / \partial \mu)=0$ and $(\partial \ln L / \partial \lambda)=0$. It is observed that MLEs cannot be obtained in explicit forms analytically from the system, so one can use a numerical method to find these MLEs numerically. Here, we use the Newton method with ABLUEs as initial guesses for finding the MLEs $\widehat{\mu}$ and $\hat{\lambda}$.

\section{Fisher Information Matrix}

In this section, we develop the interval estimates for the parameters $\mu$ and $\lambda$ of the APE distribution. To see this, we derive the Fisher information matrix and then derive the asymptotic Variance-Covariance matrix of these MLEs. Hence, we have to derive the second derivatives of the $\ln L$ functions with respect to $\mu$ and $\lambda$. From equations (7) and (8), we have

$$
\begin{aligned}
& \frac{\partial^{2} \ln L}{\partial \mu^{2}}=-\lambda^{2} \ln \alpha \sum_{i=1}^{m} \frac{e^{-\lambda\left(x_{i}-\mu\right)}\left(\left(\alpha^{e^{-\lambda\left(x_{i}-\mu\right)}}-1\right)^{2}-R_{i}\left(\alpha^{e^{-\lambda\left(x_{i}-\mu\right)}}\left(1-e^{-\lambda\left(x_{i}-\mu\right)} \ln \alpha\right)-1\right)\right)}{\left(\alpha^{e^{-\lambda\left(x_{i}-\mu\right)}}-1\right)^{2}}, \\
& \frac{\partial^{2} \ln L}{\partial \lambda^{2}}=-\frac{m}{\lambda^{2}}-\ln \alpha \sum_{i=1}^{m}\left(x_{i}-\mu\right)^{2} e^{-\lambda\left(x_{i}-\mu\right)}-\ln \alpha \sum_{i=1}^{m} \frac{R_{i}\left(x_{i}-\mu\right)^{2} e^{-\lambda\left(x_{i}-\mu\right)}\left(1+\alpha^{e^{-\lambda\left(x_{i}-\mu\right)}}\left(e^{-\lambda\left(x_{i}-\mu\right)} \ln \alpha-1\right)\right)}{\left(\alpha^{e^{-\lambda\left(x_{i}-\mu\right)}}-1\right)^{2}}, \\
& \frac{\partial^{2} \ln L}{\partial \lambda \partial \mu}=m+\ln \alpha \sum_{i=1}^{m}\left(\lambda\left(x_{i}-\mu\right)-1\right) e^{-\lambda\left(\left(x_{i}-\mu\right)\right.} \\
& +\sum_{i=1}^{m} \frac{R_{i} e^{-\lambda\left(x_{i}-\mu\right)}\left(\alpha^{e^{-\lambda\left(x_{i}-\mu\right)}}\left(1-\lambda\left(x_{i}-\mu\right)\right)+\lambda\left(x_{i}-\mu\right)\left(\alpha^{e^{-\lambda\left(x_{i}-\mu\right)}} e^{-\lambda\left(x_{i}-\mu\right)} \ln \alpha+1\right)-1\right)}{\left(\alpha^{e^{-\lambda\left(x_{i}-\mu\right)}}-1\right)^{2}} .
\end{aligned}
$$

From equations (9)-(11), the observed information matrix can be inverted to obtain the asymptotic variancecovariance matrix of the MLEs as follows:

$$
\left[\begin{array}{c}
-\left.\frac{\partial^{2} \ln L}{\partial \mu^{2}}\right|_{\widehat{\mu}, \widehat{\lambda}}-\left.\frac{\partial^{2} \ln L}{\partial \mu \partial \lambda}\right|_{\widehat{\mu}, \widehat{\lambda}} \\
-\left.\frac{\partial^{2} \ln L}{\partial \mu \partial \lambda}\right|_{\widehat{\mu}, \widehat{\lambda}}-\left.\frac{\partial^{2} \ln L}{\partial \lambda^{2}}\right|_{\widehat{\mu}, \widehat{\lambda}}
\end{array}\right]^{-1} \cdot
$$

The distribution of pivotal quantities

$$
\begin{aligned}
& P_{1}=\frac{\widehat{\mu}-\mu}{\sqrt{\operatorname{Var}(\hat{\mu})}}, \\
& P_{2}=\frac{\hat{\lambda}-\lambda}{\sqrt{\operatorname{Var}(\hat{\lambda})}},
\end{aligned}
$$

is considered to be standard normal regarded to the asymptotic properties of MLEs. From $P_{1}$ and $P_{2}$, one can construct the confidence interval for $\mu$ and $\lambda$, respectively.
Hence, 95\% confidence intervals for $\mu$ and $\lambda$ based on the MLEs are given by

$$
\begin{aligned}
& {[\widehat{\mu}-1.96 \sqrt{\operatorname{Var}(\widehat{\mu})}, \widehat{\mu}+1.96 \sqrt{\operatorname{Var}(\widehat{\mu})}],} \\
& {[\widehat{\lambda}-1.96 \sqrt{\operatorname{Var}(\widehat{\lambda})}, \hat{\lambda}+1.96 \sqrt{\operatorname{Var}(\widehat{\lambda})}] .}
\end{aligned}
$$

\section{Approximate Best Linear Unbiased Estimator}

The BLUEs for $\mu$ and $\lambda$ are so difficult due to the difficulty in solving the variance-covariance matrix $\Sigma$ for the APE distribution and also its inverse $\Sigma^{-1}$ for large sample. In order to that we derive the ABLUEs of $\mu$ and $\lambda$. To do this, suppose that the progressively Type-II censored sample

$$
X_{1: m: n}^{\left(R_{1}, \ldots, R_{m}\right)}, X_{2: m: n}^{\left(R_{1}, \ldots, R_{m}\right)}, \ldots, X_{m: m: n}^{\left(R_{1}, \ldots, R_{m}\right)},
$$

has been taken from APE distribution. By following the method described by Balakrishnan and Aggarwala [2], we compute the ABLUEs $\mu^{*}$ and $\lambda^{*}$, respectively, as follows: 


$$
\begin{aligned}
& \mu^{*}=\sum_{i=1}^{m} A_{i} X_{i: m: n}^{\left(R_{1}, \ldots, R_{m}\right)}, \\
& \lambda^{*}=\sum_{i=1}^{m} B_{i} X_{i: m: n}^{\left(R_{1}, \ldots, R_{m}\right)},
\end{aligned}
$$

where $A_{i}$ and $B_{i}$ are the coefficients of the ABLUEs. In order to find these coefficients, we developed an $R$-Code program. The values of $A_{i}$ and $B_{i}$ are given in Table 1 and checked for the conditions:

$$
\begin{aligned}
& \sum_{i=1}^{m} A_{i} \simeq 1, \\
& \sum_{i=1}^{m} B_{i} \simeq 0,
\end{aligned}
$$

which is achieved. For more about the ABLUEs and the coefficients $A_{i}$ and $B_{i}$, see Balakrishnan and Aggarwala [2] page 81 to page 110 .

\section{Simulation Study and Numerical Calculations}

Following the algorithm presented by Balakrishnan and Sandhu [12], one can generate progressively Type-II censored samples from the standard APE distribution taking $\mu=0$ and $\lambda=1$. We apply the given algorithm below to perform the numerical computations and simulations proposed in the previous sections.

Step 1: $m$ independent uniform $(0,1)$ variables $W_{1}, \ldots$, $W_{m}$ are generated

Step 2: for $\left(R_{1}, \ldots, R_{m}\right)$ progressively censoring scheme, set $V_{i}=W_{i}^{1 /\left(i+\sum_{j=m-i+1}^{m} R_{i}\right)}$ for $i=1,2, \ldots, m$

Step 3: set $U_{i}=1-V_{m} V_{m-1} \ldots V_{m-i+1}$ for $i=1,2, \ldots$, $m$, then $U_{i: m: n}, \ldots, U_{m: m: n}$ is progressively Type-II censored sample of size $m$ from uniform $(0,1)$
Step 4: finally, for some given values of $\mu$ and $\lambda$, we have $X_{i}=\mu+1 / \lambda \log \left[\log \alpha / \log \alpha-\log \left[1+(\alpha-1) u_{i}\right]\right]$ progressively generated from APE distribution

After applying the algorithm and selecting sample sizes and censoring schemes from APE distribution given in Table 2, we compute the ABLUEs of $\mu$ and $\lambda$ from equations (16) and (17) and tabulate it in Table 1 for some chosen values of the shape parameter $\alpha=1.5,2.5,3.5$. We also compute the biases and mean square error (MSE) of the ABLUEs of $\mu$ and $\lambda$ in Tables 3-5. As we mentioned before, we use the ABLUEs of $\mu$ and $\lambda$ to estimate the MLEs of $\mu$ and $\lambda$; these MLEs, biases, and MSEs of the parameters are tabulated in Tables 6-8. All the computations are computed using $R$ program over 5000 Monte Carlo simulations. The computations are carried out for some selected samples size: $n=5,10,20,30,50,80,100$.

Example 1. From the APE distribution with $\mu=0, \lambda=1$, and $\alpha=2.5$, we generate a progressively Type-II censored sample of size $m=6$, from $n=20$. The progressive censoring scheme and its observations are

\begin{tabular}{ccccccc}
\hline$i$ & 1 & 2 & 3 & 4 & 5 & 6 \\
\hline$x_{i}$ & 0.1992 & 0.2402 & 0.4857 & 0.4920 & 0.5762 & 1.1805 \\
\hline$R_{i}$ & 2 & 0 & 0 & 8 & 0 & 4 \\
\hline
\end{tabular}

Using the above censored sample and from the likelihood equations, (7) and (8), we compute the MLEs of $\mu$ and $\lambda$ to be

$$
\begin{aligned}
& \widehat{\mu}=-0.1764, \\
& \widehat{\lambda}=1.0982 .
\end{aligned}
$$

Using the above MLEs, the 95\% confidence intervals for the location $\mu$ and scale $\lambda$ parameters are $(-0.4812,2.2661)$ and $(1.0255,2.3089)$, respectively.

Similarly, we obtain the ABLUEs of $\mu$ and $\lambda$ from Table 1 and equations (16) and (17) to be

$$
\begin{aligned}
& \mu^{*}=0.1992 * 1.1633+0.2402 * 0.0004+0.4857 *(-0.0019)+0.4920 *(-0.0800)+0.5762 *(-0.0082)+1.1805 *(-0.0736) \\
& \mu^{*}=0.0999 \\
& \lambda^{*}=0.1992 *(-2.1928)+0.2402 * 0.0467+0.4857 * 0.0674+0.4920 * 1.0560+0.5762 * 0.1279+1.1805 * 0.8948 \\
& \lambda^{*}=1.2568
\end{aligned}
$$

Here, we notice that the ABLUEs are so closed to the MLEs of the parameters $\mu$ and $\lambda$, and this shows that ABLUEs give good initial guesses when estimating the MLEs of these parameters.

Example 2. The following data $\{2.247,2.64,2.908,3.099$, $3.126,3.245,3.328,3.355,3.383,3.572,3.581,3.681,3.726$, $3.727,3.728,3.783,3.785,3.786,3.896,3.912,3.964,4.05$, $4.063,4.082,4.111,4.118,4.141,4.246,4.251,4.262,4.326$,
$4.402,4.457,4.466,4.519,4.542,4.555,4.614,4.632,4.634$, 4.636, 4.678, 4.698, 4.738, 4.832, 4.924, 5.043, 5.099, 5.134, $5.359,5.473,5.571,5.684,5.721,5.98,6.06\}$ was taken from Bader and Priest [13] and represents the strength for single carbon fibers and impregnated 1000-cardon fiber tows, measured in GPa. The data is reported at gange length $1 \mathrm{~mm}$. These data fitted the APE distribution, see Mahdavi and Kundu [11]. We found that the MLEs of $\mu$ and $\lambda$ for the complete data are 
TABLE 1: Coefficients of ABLUEs of $\mu$ and $\lambda$ from APE distribution.

\begin{tabular}{|c|c|c|c|c|c|c|}
\hline \multirow{2}{*}{ Scheme/sum } & \multicolumn{2}{|c|}{$\alpha=1.5$} & \multicolumn{2}{|c|}{$\alpha=2.5$} & \multicolumn{2}{|c|}{$\alpha=3.5$} \\
\hline & $A_{i}$ & $B_{i}$ & $A_{i}$ & $B_{i}$ & $A_{i}$ & $B_{i}$ \\
\hline \multirow{3}{*}{ [1] } & 1.3300 & -1.5176 & 1.3522 & -1.3062 & 1.3716 & -1.2033 \\
\hline & -0.0823 & 0.4036 & -0.0625 & 0.3028 & -0.0457 & 0.2546 \\
\hline & -0.2477 & 1.1140 & -0.2898 & 1.0034 & -0.3259 & 0.9487 \\
\hline Sum & $\approx 1$ & $\approx 0$ & $\approx 1$ & $\approx 0$ & $\approx 1$ & $\approx 0$ \\
\hline \multirow{5}{*}{ [2] } & 1.1091 & -0.9709 & 1.1117 & -0.8253 & 1.1368 & -0.7565 \\
\hline & -0.0186 & 0.1851 & -0.0079 & 0.1225 & 0.0022 & 0.0939 \\
\hline & -0.0235 & 0.2153 & -0.0220 & 0.1803 & -0.0209 & 0.1655 \\
\hline & -0.0277 & 0.2416 & -0.0324 & 0.2204 & -0.0366 & 0.2101 \\
\hline & -0.0393 & 0.3289 & -0.0494 & 0.3022 & -0.0584 & 0.2870 \\
\hline Sum & $\approx 1$ & $\approx 0$ & $\approx 1$ & $\approx 0$ & $\approx 1$ & $\approx 0$ \\
\hline \multirow{6}{*}{ [3] } & 1.1651 & -2.7948 & 1.1633 & -2.1928 & 1.1613 & -1.8893 \\
\hline & -0.0064 & 0.1219 & 0.0004 & 0.0467 & 0.0076 & 0.0078 \\
\hline & -0.0070 & 0.1305 & -0.0019 & 0.0674 & 0.0034 & 0.0368 \\
\hline & -0.0805 & 1.3575 & -0.0800 & 1.0560 & -0.0802 & 0.9056 \\
\hline & -0.0094 & 0.1659 & -0.0082 & 0.1279 & -0.0071 & 0.1115 \\
\hline & -0.0618 & 1.0190 & -0.0736 & 0.8948 & -0.0850 & 0.8275 \\
\hline Sum & $\approx 1$ & $\approx 0$ & $\approx 1$ & $\approx 0$ & $\approx 1$ & $\approx 0$ \\
\hline \multirow{8}{*}{ [4] } & 1.0831 & -2.1149 & 1.0770 & -1.6014 & 1.0719 & -1.3558 \\
\hline & -0.0419 & 1.0740 & -0.0331 & 0.7273 & -0.0254 & 0.5638 \\
\hline & -0.0036 & 0.0980 & -0.0014 & 0.0565 & 0.0007 & 0.0383 \\
\hline & -0.0041 & 0.1085 & -0.0030 & 0.0784 & -0.0021 & 0.0662 \\
\hline & -0.0045 & 0.1176 & -0.0043 & 0.0958 & -0.0043 & 0.0871 \\
\hline & -0.0049 & 0.1254 & -0.0054 & 0.1094 & -0.0060 & 0.1026 \\
\hline & -0.0053 & 0.1320 & -0.0063 & 0.1198 & -0.0073 & 0.1137 \\
\hline & -0.0189 & 0.4594 & -0.0234 & 0.4141 & -0.0276 & 0.3870 \\
\hline Sum & $\approx 1$ & $\approx 0$ & $\approx 1$ & $\approx 0$ & $\approx 1$ & $\approx 0$ \\
\hline \multirow{10}{*}{ [5] } & 1.1089 & -4.4962 & 1.1086 & -3.4660 & 1.1079 & -2.9249 \\
\hline & -0.0015 & 0.0665 & 0.0003 & 0.0163 & 0.0023 & -0.0116 \\
\hline & -0.0015 & 0.0685 & 0.0001 & 0.0216 & 0.0018 & -0.0037 \\
\hline & -0.0016 & 0.0704 & -0.0002 & 0.0267 & 0.0014 & 0.0037 \\
\hline & -0.0016 & 0.0723 & -0.0004 & 0.0315 & 0.0010 & 0.0107 \\
\hline & -0.0018 & 0.0742 & -0.0006 & 0.0362 & 0.0006 & 0.0173 \\
\hline & -0.0017 & 0.0760 & -0.0007 & 0.0407 & 0.0003 & 0.0235 \\
\hline & -0.0018 & 0.0778 & -0.0009 & 0.0449 & -0.0004 & 0.0294 \\
\hline & -0.0018 & 0.0796 & -0.0011 & 0.0490 & -0.0004 & 0.0349 \\
\hline & -0.0957 & 3.9109 & -0.1056 & 3.1991 & -0.1149 & 2.8206 \\
\hline Sum & $\approx 1$ & $\approx 0$ & $\approx 1$ & $\approx 0$ & $\approx 1$ & $\approx 0$ \\
\hline \multirow{15}{*}{ [6] } & 1.0393 & -2.5953 & 1.0390 & -2.0121 & 1.0386 & -1.7159 \\
\hline & -0.0006 & 0.0416 & 0.0001 & 0.0097 & 0.0009 & -0.0069 \\
\hline & -0.0006 & 0.0430 & 0.0000 & 0.0133 & 0.0007 & -0.0017 \\
\hline & -0.0006 & 0.0444 & 0.0000 & 0.0168 & 0.0005 & 0.0032 \\
\hline & S1002007120304962 & 0.0457 & -0.0001 & 0.0200 & 0.0004 & 0.0078 \\
\hline & -0.0007 & 0.0470 & -0.0002 & 0.0231 & 0.0002 & 0.0120 \\
\hline & -0.0007 & 0.0483 & -0.0003 & 0.0261 & 0.0001 & 0.0160 \\
\hline & -0.0007 & 0.0495 & -0.0004 & 0.0289 & 0.0001 & 0.0198 \\
\hline & -0.0007 & 0.0507 & -0.0004 & 0.0316 & -0.0002 & 0.0233 \\
\hline & -0.0008 & 0.0519 & -0.0005 & 0.0342 & -0.0003 & 0.0266 \\
\hline & -0.0008 & 0.0531 & -0.0006 & 0.0366 & -0.0004 & 0.0297 \\
\hline & -0.0008 & 0.0542 & -0.0006 & 0.0389 & -0.0005 & 0.0326 \\
\hline & -0.0008 & 0.0552 & -0.0007 & 0.0411 & -0.0006 & 0.0353 \\
\hline & -0.0008 & 0.0563 & -0.0008 & 0.0433 & -0.0007 & 0.0379 \\
\hline & -0.0301 & 1.9544 & -0.0345 & 1.6485 & -0.0388 & 1.4803 \\
\hline Sum & $\approx 1$ & $\approx 0$ & $\approx 1$ & $\approx 0$ & $\approx 1$ & $\approx 0$ \\
\hline
\end{tabular}


TABle 1: Continued.

\begin{tabular}{|c|c|c|c|c|c|c|}
\hline \multirow{2}{*}{ Scheme/sum } & \multicolumn{2}{|c|}{$\alpha=1.5$} & \multicolumn{2}{|c|}{$\alpha=2.5$} & \multicolumn{2}{|c|}{$\alpha=3.5$} \\
\hline & $A_{i}$ & $B_{i}$ & $A_{i}$ & $B_{i}$ & $A_{i}$ & $B_{i}$ \\
\hline \multirow{20}{*}{ [7] } & 1.0405 & -3.3421 & 1.0388 & -2.5137 & 1.0370 & -2.0908 \\
\hline & -0.0099 & 0.8228 & -0.0082 & 0.5501 & -0.0066 & 0.4091 \\
\hline & -0.0003 & 0.0309 & 0.0008 & 0.0079 & 0.0005 & -0.0040 \\
\hline & -0.0003 & 0.0317 & 0.0003 & 0.0099 & 0.0005 & -0.0011 \\
\hline & -0.0004 & 0.0325 & 0.0000 & 0.0118 & 0.0004 & 0.0016 \\
\hline & -0.0004 & 0.0332 & -0.0004 & 0.0136 & 0.0003 & 0.0048 \\
\hline & -0.0004 & 0.0339 & -0.0008 & 0.0154 & 0.0002 & 0.0067 \\
\hline & -0.0004 & 0.0347 & -0.0001 & 0.0171 & 0.0002 & 0.0090 \\
\hline & -0.0004 & 0.0354 & -0.0002 & 0.0188 & 0.0000 & 0.0112 \\
\hline & -0.0004 & 0.0361 & -0.0002 & 0.0204 & 0.0000 & 0.0133 \\
\hline & -0.0004 & 0.0367 & -0.0002 & 0.0219 & 0.0000 & 0.0154 \\
\hline & -0.0004 & 0.0374 & -0.0003 & 0.0234 & 0.0000 & 0.0173 \\
\hline & -0.0004 & 0.0381 & -0.0003 & 0.0248 & -0.0001 & 0.0191 \\
\hline & -0.0004 & 0.0387 & -0.0003 & 0.0262 & -0.0002 & 0.0209 \\
\hline & -0.0005 & 0.0393 & -0.0003 & 0.0275 & -0.0002 & 0.0225 \\
\hline & -0.0005 & 0.0399 & -0.0004 & 0.0288 & -0.0003 & 0.0241 \\
\hline & -0.0005 & 0.0405 & -0.0004 & 0.0300 & -0.0003 & 0.0257 \\
\hline & -0.0005 & 0.0411 & -0.0004 & 0.0312 & -0.0004 & 0.0271 \\
\hline & -0.0115 & 0.9314 & -0.0130 & 0.7792 & -0.0146 & 0.6968 \\
\hline & -0.0125 & 1.0078 & -0.0144 & 0.8557 & -0.0164 & 0.7713 \\
\hline Sum & $\approx 1$ & $\approx 0$ & $\approx 1$ & $\approx 0$ & $\approx 1$ & $\approx 0$ \\
\hline
\end{tabular}

TABLE 2: Sample sizes and censoring schemes from APE distribution.

\begin{tabular}{lccc}
\hline$n$ & $m$ & Scheme & Censoring scheme \\
\hline 5 & 3 & {$[1]$} & $(1,0,1)$ \\
10 & 5 & {$[2]$} & $(5,0,0,0,0)$ \\
20 & 6 & {$[3]$} & $(2,0,0,8,0,4)$ \\
30 & 8 & {$[4]$} & $(10,10,0,0,0,0,0,2)$ \\
50 & 10 & {$[5]$} & $(0,0,0,0,0,0,0,0,0,40)$ \\
80 & 15 & {$[6]$} & $(35,0,0,0,0,0,0,0,0,0,0,0,0,0,30)$ \\
100 & 20 & {$[7]$} & $(20,20,0,0,0,0,0,0,0,0,0,0,0,0,0,0,0,0,20,20)$ \\
\hline
\end{tabular}

TABLE 3: ABLUEs $\mu^{*}, \lambda^{*}$, biases, and MSEs for $\mu$ and $\lambda$ from APE distribution when $\alpha=1.5$.

\begin{tabular}{lccccc}
\hline Scheme & $\mu^{*}$ & $\lambda^{*}$ & $\operatorname{Bias}\left(\mu^{*}\right)$ & $\operatorname{Bias}\left(\lambda^{*}\right)$ & $\operatorname{MSE}\left(\mu^{*}\right)$ \\
\hline$[1]$ & 0.0477 & 1.0575 & 0.0059 & 0.1439 & 0.0736 \\
{$[2]$} & 0.1347 & 1.2711 & 0.0036 & 0.1172 & 0.0178 \\
{$[3]$} & -0.0088 & 1.2199 & 0.0057 & 0.0098 & 0.0043 \\
{$[4]$} & -0.0376 & 1.3769 & 0.0026 & 0.0694 & 0.0021 \\
{$[5]$} & -0.0190 & 1.4204 & 0.0023 & 0.0177 & 0.0006 \\
{$[6]$} & -0.0090 & 1.0030 & 0.0090 & 0.0030 & 0.0005 \\
{$[7]$} & -0.0118 & 1.1481 & 0.0008 & 0.0097 & 0.0001 \\
\hline
\end{tabular}

TABle 4: ABLUEs $\mu^{*}, \lambda^{*}$, biases, and MSEs for $\mu$ and $\lambda$ from APE distribution when $\alpha=2.5$.

\begin{tabular}{lccccc}
\hline Scheme & $\mu^{*}$ & $\lambda^{*}$ & $\operatorname{Bias}\left(\mu^{*}\right)$ & $\operatorname{Bias}\left(\lambda^{*}\right)$ & $\operatorname{MSE}\left(\mu^{*}\right)$ \\
\hline$[1]$ & -0.1913 & 1.3600 & 0.0256 & 0.1536 & 0.1143 \\
{$[2]$} & -0.1382 & 1.4843 & 0.0124 & 0.2085 & 0.0347 \\
{$[3]$} & -0.0803 & 1.3727 & 0.0019 & 0.0037 & 0.0071 \\
{$[4]$} & -0.0422 & 1.3733 & 0.0072 & 0.0755 & 0.0028 \\
{$[5]$} & -0.0280 & 1.2874 & 0.0004 & 0.0233 & 0.0013 \\
{$[6]$} & -0.0150 & 0.9950 & 0.0004 & 0.0022 & 0.0003 \\
{$[7]$} & 0.0044 & 1.1136 & 0.0005 & 0.0009 & 0.1754 \\
& & & & & 0.0002 \\
\hline
\end{tabular}


TABle 5: ABLUEs $\mu^{*}, \lambda^{*}$, biases, and MSEs for $\mu$ and $\lambda$ from APE distribution when $\alpha=3.5$.

\begin{tabular}{lccccc}
\hline Scheme & $\mu^{*}$ & $\lambda^{*}$ & $\operatorname{Bias}\left(\mu^{*}\right)$ & $\operatorname{Bias}\left(\lambda^{*}\right)$ & $\operatorname{MSE}\left(\mu^{*}\right)$ \\
\hline$[1]$ & -0.0484 & 1.2976 & 0.0615 & 0.2154 & 0.1817 \\
{$[2]$} & -0.2212 & 1.2530 & 0.0394 & 0.1533 & 0.0439 \\
{$[3]$} & -0.1096 & 1.3558 & 0.0054 & 0.0131 & 0.0105 \\
{$[4]$} & -0.0761 & 1.4350 & 0.0135 & 0.0537 & 0.0030 \\
{$[5]$} & -0.0105 & 1.4435 & 0.0039 & 0.0132 & 0.0016 \\
{$[6]$} & 0.0484 & 0.9950 & 0.0010 & 0.0146 & 0.03248 \\
{$[7]$} & 0.0009 & 1.1870 & 0.0011 & 0.0008 & 0.1692 \\
\hline
\end{tabular}

TABle 6: MLEs $\widehat{\mu}, \hat{\lambda}$, biases, and MSEs for $\mu$ and $\lambda$ from APE distribution when $\alpha=1.5$.

\begin{tabular}{|c|c|c|c|c|c|c|}
\hline Scheme & $\widehat{\mu}$ & $\hat{\lambda}$ & $\operatorname{Bias}(\widehat{\mu})$ & $\operatorname{Bias}(\hat{\lambda})$ & $\operatorname{MSE}(\widehat{\mu})$ & $\operatorname{MSE}(\widehat{\lambda})$ \\
\hline [1] & -0.0822 & 1.3960 & 0.0140 & 0.0008 & 0.0195 & 0.0001 \\
\hline [2] & -0.0836 & 1.0989 & 0.0110 & 0.0008 & 0.0121 & 0.0001 \\
\hline [3] & -0.0931 & 1.1621 & 0.0116 & 0.0009 & 0.0135 & 0.0002 \\
\hline [4] & -0.0100 & 1.0914 & 0.0110 & 0.0010 & 0.0119 & 0.0000 \\
\hline [5] & -0.0748 & 0.9860 & 0.0098 & 0.0007 & 0.0097 & 0.0000 \\
\hline [6] & -0.0560 & 1.0600 & 0.0030 & 0.0005 & 0.0090 & 0.0000 \\
\hline [7] & -0.0611 & 1.1480 & 0.0011 & 0.0000 & 0.0013 & 0.0000 \\
\hline
\end{tabular}

TABle 7: MLEs $\widehat{\mu}, \hat{\lambda}$, biases, and MSEs for $\mu$ and $\lambda$ from APE distribution when $\alpha=2.5$.

\begin{tabular}{lcccccc}
\hline Scheme & $\widehat{\mu}$ & $\widehat{\lambda}$ & $\operatorname{Bias}(\widehat{\mu})$ & $\operatorname{Bias}(\widehat{\lambda})$ & $\operatorname{MSE}(\widehat{\mu})$ & 0.0132 \\
\hline$[1]$ & -0.0533 & 1.1493 & 0.0115 & 0.0005 & 0.0131 & 0.0001 \\
{$[2]$} & -0.1205 & 1.1430 & 0.0111 & 0.0012 & 0.0152 \\
{$[3]$} & -0.1896 & 1.2341 & 0.0123 & 0.0019 & 0.0002 \\
{$[4]$} & -0.1870 & 1.1838 & 0.0118 & 0.0018 & 0.0004 \\
{$[5]$} & -0.1703 & 1.2174 & 0.0122 & 0.0017 & 0.0003 \\
{$[6]$} & -0.1670 & 1.1560 & 0.0020 & 0.0002 & 0.0046 \\
{$[7]$} & -0.1140 & 1.1236 & 0.0011 & 0.0001 & 0.0012 \\
\hline
\end{tabular}

TABle 8: MLEs $\hat{\mu}, \hat{\lambda}$, biases, and MSEs for $\mu$ and $\lambda$ from APE distribution when $\alpha=3.5$.

\begin{tabular}{|c|c|c|c|c|c|c|}
\hline Scheme & $\widehat{\mu}$ & $\widehat{\lambda}$ & $\operatorname{Bias}(\widehat{\mu})$ & $\operatorname{Bias}(\widehat{\lambda})$ & $\operatorname{MSE}(\widehat{\mu})$ & $\operatorname{MSE}(\widehat{\lambda})$ \\
\hline$[1]$ & -0.1334 & 1.1840 & 0.0118 & 0.0013 & 0.0140 & 0.0002 \\
\hline [2] & -0.0930 & 1.0147 & 0.0101 & 0.0009 & 0.0102 & 0.0001 \\
\hline [3] & -0.1938 & 1.0048 & 0.0120 & 0.0019 & 0.0145 & 0.0003 \\
\hline [4] & -0.2411 & 1.1920 & 0.0120 & 0.0024 & 0.0142 & 0.0005 \\
\hline [5] & -0.1666 & 1.0449 & 0.0110 & 0.0017 & 0.0109 & 0.0003 \\
\hline [6] & -0.2242 & 1.0345 & 0.0020 & 0.0002 & 0.0040 & 0.0000 \\
\hline [7] & -0.1478 & 1.0500 & 0.0015 & 0.0001 & 0.0024 & 0.0000 \\
\hline
\end{tabular}

$$
\begin{aligned}
& \widehat{\mu}=2.3655, \\
& \widehat{\lambda}=1.2571 .
\end{aligned}
$$

Using the above MLEs, the 95\% confidence intervals for the location $\mu$ and scale $\lambda$ parameters are $(0.9453,1.5688)$ and $(2.1225,2.6085)$, respectively. Comparing the results in this paper to that in Mahdavi and Kundu [11], we see that these results are so close to each other.

\section{Conclusion}

The MLEs and ABLUEs of the location and scale parameters from APE distribution are derived and tabulated for some selected sample size and shape parameter. The ABLUEs are so closed to the MLEs which gives good initial guesses in estimating MLEs. An $R$ code program was performed to find the ABLUEs and MLEs. It will be interesting to study the Type-I and Type-II hybrid censoring scheme from the APE distribution in future And also Bayesian and non-Bayesian inference under adaptive type-II progressive censored sample from APE distribution.

\section{Data Availability}

The data used to support the findings of this study are included within the article.

\section{Conflicts of Interest}

No potential conflicts of interest were reported by the author. 


\section{Acknowledgments}

The author extends his appreciation to the Deanship of Scientific Research at Majmaah University for funding this work under project number 91/38.

\section{References}

[1] M. Salah, "Moments from progressive type-II censored data of Marshall-Olkin exponential distribution," Journal of Applied Mathematical Research, vol. 1, no. 4, pp. 771-786, 2012.

[2] N. Balakrishnan and R. Aggarwala, Progressive Censoring: Theory, Method and Applications, Birkhauser, Basel, Switzerland, 2000.

[3] B. Pradhan and D. Kundu, "On progressively censored generalized exponential distribution," Test, vol. 18, no. 3, pp. 497-515, 2009.

[4] C.-T. Lin, S. J. S. Wu, and N. Balakrishnan, "Inference for loggamma distribution based on progressively type-II censored data," Communications in Statistics-Theory and Methods, vol. 35, no. 7, pp. 1271-1292, 2006.

[5] N. Balakrishnan, "Progressive censoring methodology: an appraisal," Test, vol. 16, no. 2, pp. 211-259, 2007.

[6] N. Balakrishnan, E. Cramer, U. Kamps, and N. Schenk, "Progressive type II censored order statistics from exponential distributions," Statistics, vol. 35, no. 4, pp. 537-556, 2001.

[7] M. Salah, "Moments of upper record values from marshallolkin exponential distribution," Journal of Statistics Applications \& Probability, vol. 5, no. 2, pp. 1-7, 2016.

[8] M. Salah, "Bayesian estimation of the scale parameter of the Marshall-Olkin exponential distribution under progressively type-II censored samples," Journal of Statistical Theory and Applications, vol. 17, no. 1, pp. 1-14, 2018.

[9] M. Salah, "Parameter estimation of the Marshall-Olkin exponential distribution under type-II hybrid censoring schemes and its applications," Journal of Statistics Applications \& Probability, vol. 5, no. 3, pp. 1-8, 2016.

[10] H. M. R. Khan, S. B. Provost, and A. Singh, "Predictive inference from a two-parameter Rayleigh life model given a doubly censored sample," Communications in StatisticsTheory and Methods, vol. 39, no. 7, pp. 1237-1246, 2010.

[11] A. Mahdavi and D. Kundu, "A new method for generating distributions with an application to exponential distribution," Communications in Statistics-Theory and Methods, vol. 46, no. 13, pp. 6543-6557, 2017.

[12] N. Balakrishnan and R. A. Sandhu, "A simple simulational algorithm for generating progressive type-II censored samples," The American Statistician, vol. 49, no. 2, pp. 229-230, 1995.

[13] M. Bader and A. Priest, Statistical Aspects of Fibre and Bundle Strength in Hybrid Composites, Progress in Science and Engineering of Composites, Springer, Berlin, Germany, 1982. 